\title{
Acknowledgement to Reviewers of Antioxidants in 2018
}

Antioxidants Editorial Office

MDPI, St. Alban-Anlage 66, 4052 Basel, Switzerland

Published: 9 January 2019

Rigorous peer-review is the corner-stone of high-quality academic publishing. The editorial team greatly appreciates the reviewers who contributed their knowledge and expertise to the journal's editorial process over the past 12 months. In 2018, a total of 189 papers were published in the journal, with a median time to first decision of 15 days and a median time to publication of 40 days. The editors would like to express their sincere gratitude to the following reviewers for their cooperation and dedication in 2018:

Abrahamsson, Thomas

Adam, Vojtěch

Afolayan, Adeleye J.

Åkerström, Bo

Almajano, María Pilar

Altemimi, Ammar

Amaya, Enrique

Amoaku, Winfried M.

Amorós, Asunción

Angelino, Donato

Ansorena, Diana

Antognelli, Cinzia

Arguelles, Sandro

Ariga, Katsuhiko

Armand, Martine

Audi, Said

Avato, Pinarosa

Avila-Román, Javier

Aye, Yimon

Aykin-burns, Nukhet

Bachschmid, Markus Michael

Baker, Julien

Baldisserotto, Anna

Ballmann, Christopher

Banerjee, Kalpita

Barba, Francisco J.

Barbagallo, Davide
Barbaric, Monika

Barbe, Mary F.

Bárcena, José Antonio

Bartlett, David B.

Battaglino, Ricardo

Bauer, Georg

Beharry, Kay D.

Bellés, José María

Bellner, Lars

Bendich, Adrianne

Beresford, Tom

Bernini, Roberta

Blumberg, Jeffrey

Boggia, Raffaella

Böhm, Volker

Bonomini, Francesca

Borer, Katarina $\mathrm{T}$.

Boschi-Muller, Sandrine

Brash, Alan

Brewer, Alison C.

Brodzki, Piotr

Bubb, Kristen J.

Buchanan, Bob

Budryn, Grażyna

Cairrão, Elisa

Calani, Luca

Campbell, Fiona M. 
Canabady-Rochelle, Laetitia L.S.

Canini, Antonella

Caradonna, Fabio

Cardoso, Susana

Carey, Amanda

Carnevale, Roberto

Carocho, Marcio

Carr, Anitra

Carvalho, Daniel

Castro-Caldas, Margarida

Cavallaro, Giacomo

Cejudo, Francisco J.

Chaiswing, Luksana

Chakraborty, Mallinath

Chang, Hsueh-Wei

Chao, Wen-Wan

Chatterjee, Jhinuk

Chen, Lin

Chen, Min

Chen, Yung-Hsiang

Cherkaoui-Malki, Mustapha

Cherniack, Evan Paul

Chiang, Hsiu-Mei

Chilibeck, Phil

Chiu, Joyce

Chobot, Vladimir

Chohan, Magali

Christides, Tatiana

Ciaccio, Marcello

Çiçek, Serhat Sezai

Ciofani, Gianni

Ciofi-Baffoni, Simone

Cione, Erika

Čipak Gašparović, Ana

Coleman, Mitchell C.

Contino, Marialessandra

Corino, Carlo

Costa, Lucio G.

Craige, Siobhan M.

Cuffe, James

Cumming, Kristoffer T.

Da Silva, Robin P.

Dachs, Gabi
Dalessandro, Giuseppe

Dall'Acqua, Stefano

Das, Kumuda C.

Dasgupta, Santanu

David, Luminiţa

Davies, Michael

Davinelli, Sergio

De Filippis, Vincenzo

De La Vega, Laureano

De Las Heras, Javier

De Mendonça, Dina I. M. D.

De Vendittis, Emmanuele

Deli, Chariklia K.

DeSmet, Stefan

Diakowska, Dorota

Dietz, Karl-Josef

Doles, Jason D.

Donato, Rosario

Donno, Dario

Doriano Bianco, Armando

Dubrova, Yuri

Dudas, Jozsef

Düfer, Martina

Edge, Ruth

Ekiert, Halina

Elhabiri, Mourad

Emmert, Steffen

Espinosa-Diez, Cristina

Esposito, Ciro

Fan, Yichong

Fang, Ronnie H.

Feresin, Rafaela G.

Fernandes, Ana S.

Fielding, Alistair

Finno, Carrie J.

Fiorino, Ferdinando

Flack, Kyle D.

Florencio, Francisco J.

Frank, Saša

Franken, Nicolaas A. P.

Friedman, Haya

Frolov, Andrej

Fuchs, Dietmar 
Furdui, Cristina Maria

Gabai, Gianfranco

Galea, Ian

Galic, Ante

Galvano, Fabio

Gan, Renyou

Ganini, Douglas

Garcia-Castineiras, Sixto

García-Moreno, Valme

Garten, Ryan

Gasparrini, Massimiliano

Gaucher, Caroline

Geest, Bart De

Gentile, Fabrizio

Georgakilas, Alexandros

Georgopoulos, Nik

Geraghty, Patrick

Gerber, Leonard E.

Gibson, Gary E.

Gillis, Keith

Giovinazzo, Giovanna

Gizdavic-Nikolaidis, Marija

Golestaneh, Nady

Golmohamadi, Amir

Gong, Xiaoming

Gonzalez, Michael J.

González, Sonia

González-Gallego, Javier

Gorgan, Lucian

Goua, Marie

Greenberger, Joel S.

Greenlief, Michael

Grimm, Bernhard

Grimm, Marcus O. W.

Gruhlke, Martin

Grunig, Gabriele

Guido, Luis F.

Gullón, Beatriz

Haarmann-Stemmann, Thomas

Hägglund, Per Mårten

Hampel, Daniela

Hampton, Mark

Han, Jaehong
Handzlik, Jadwiga

Hardingham, Jennifer E.

Hargreaves, Iain P.

Harper, James

Harrison, Fiona

Hart, Joanne

Hatano, Tsutomu

Hempel, Nadine

Hernández-Hernández, Ángel

Hisabori, Toru

Hitosugi, Taro

Ho, Shin-Lon

Hodge, Allison

Hossain, Mohammad

$\mathrm{Hu}$, Guoli

Huang, Hao

Iametti, Stefania

Ibrahim, Salam A.

Ientile, Riccardo

Ikegami, Takahisa

Irakli, Maria

Iskandar, Michele M.

Iwaoka, Michio

Jadavji, Nafisa M.

Janda, Elzbieta

Jaradat, Nidal

Jara-Palacios, María José

Jay-Gerin, Jean-Paul

Jia, Kailiang

Johnston, Carol

Jones, Huw

Jordão, António Manuel

Kamariah, Neelagandan

Kang, Sang Won

Kang, Jae Seung

Kao, Shu-Huei

Kappus, Rebecca

Karas, Monika

Karner, Courtney

Karplus, Andrew

Kashfi, Khosrow

Kato, Takamitsu A.

Kauppinen, Anu 
Kawakita, Hidetaka

Kawasumi, Masaoki

Kaya, Alaattin

Ke, Liang-Yin

Kelley, Eric

Khan, Mahmood

Khatib, Soliman

Kim, Hwa-Young

Kim, In-Jung

Kim, Hyung Sik

Kim, Ji Yeon

Kim, Kiyoung

Kipp, Anna Patricia

Kishimoto, Yoshimi

Klar, Agnes

Klarskov, Klaus

Klimis-Zacas, Dorothy

Kocot, Joanna

Kontogiorgis, Christos

Koopman, Rene

Koshiba, Takumi

Kotula-Balak, Malgorzata

Koulen, Peter

Kowalczewski, Przemysław

Kraemer, Brian C.

Krajka-Kuźniak, Violetta

Krupa-Kozak, Urszula

Kuhn, Hartmut

Kukula-Koch, Wirginia

Kumar, Binod

Kump, David

Kundur, Avinash Reddy

Kurdowska, Anna K.

Lance, Baumgard

Latella, Giovanni

LaVoy, Emily

Le, Thu H.

Lebaron, Richard

Ledgerwood, Elizabeth C.

Lee, Yi-Jang

Lee, Tzong-Shyuan

Lee, Jae Wook

Lee, Jin-Koo
Lee, Byeong Jae

Lee, Meng-shiou

Lehmann, Christian

Leicht, Christof

Lenaz, Giorgio

Lescure, Alain

Levine, Rodney

Li, Yonghui

Lichtenberg, Dov

Liddell, Jeffrey R.

Lin, Pei-Hui

Lingner, Joachim

Liu, Jun

Loboda, Agnieszka

Logan, Margaret

Loizzo, Monica Rosa

Lorenzo Rodriguez, Jose Manuel

Lu, Jun

Lyons, Graham

Maggini, Silvia

Maier, Patrick

Mailloux, Ryan

Mali, Vishal

Malli, Roland

Mandrich, Luigi

Manjunath, Manubolu

Marchuk, Douglas A.

Marcinek, David J.

Marengo, Barbara

Marsillach, Judit

Martin, Keith

Martínez, Sidonia

Masek, Anna

Matsufuji, Hiroshi

Maupin-Furlow, Julie

Maxwell, Jessie R.

McBean, Gethin J.

McDaneld, Tara G.

McKenzie, Mike

McPherson, Nicole O.

Mears, Jason

Mendonca, Antonio

Meng, Xiaoli 


\begin{tabular}{|c|c|}
\hline Messina, Samantha & Panagiotidis, Mihalis \\
\hline Meyer, Andreas & Pappas, Christos \\
\hline Mézes, Miklós & Park, Sunghun \\
\hline Michels, Alexander J. & Parsonage, Derek \\
\hline Miguel, Maria & Patrice, Thierry \\
\hline Miller, Yury & Pedan, Vasilisa \\
\hline Minetti, Giampaolo & Peng, Ying-Jie \\
\hline Miranda, Jose M. & Pennock, Nathan \\
\hline Mita, Giovanni & Pereira-da-Silva, Luis \\
\hline Mitra, Katsuri & Perez Gregorio, Maria Rosa \\
\hline Molina-Montes, Esther & Peskin, Alexander \\
\hline Monore, Jon & Petrou, Athinoula L. \\
\hline Montenegro, Lucia & Petruzzella, Vittoria \\
\hline Mookerjee, Shona & Pick, Edgar \\
\hline Moreno, Juan J. & Pignitter, Marc \\
\hline Moro, Loredana & Piluso, Susanna \\
\hline Morton, David & Pina Pérez, Maria Consuelo \\
\hline Motohashi, Ken & Pinto, Diana C. G. A. \\
\hline Mulas, Maurizio & Piomboni, Paola \\
\hline Müller-Schüssele, Stefanie & Plastina, Pierluigi \\
\hline Mullins, David W. & Plotnikov, Egor \\
\hline Muzykantov, Vladimir & Pochet, Roland \\
\hline Naftalin, Richard & Poplawski, Tomasz \\
\hline Nagy, Peter & Poulsen, Henrik Enghusen \\
\hline Nair, Vimal & Prentice, Howard \\
\hline Nakamaru-Ogiso, Eiko & Proestos, Charalampos \\
\hline Nawirska-Olszańska, Agnieszka & Quinzii, Catarina Maria \\
\hline Neilson, Andrew & Rachek, Lyudmila \\
\hline Nguyen, Duc Thanh & Rajauria, Gaurav \\
\hline Nikkanen, Lauri & Ramachandran, Anup \\
\hline Nomikos, Tzortzis & Ramaraj, Pandurangan \\
\hline Ohnishi, Masatoshi & Rambold, Angelika S. \\
\hline Ohta, Shinji & Rampon, Christine \\
\hline Olejar, Kenneth J. & Ranzato, Elia \\
\hline Oliveira, Rui & Rapeanu, Gabriela \\
\hline Oniga, Ilioara & Rayner, Ben \\
\hline Orian, Laura & Reddy, Hemachandra \\
\hline Oudemans-van Straaten, Heleen & Reukov, Vladimir \\
\hline Pace, Paul & Revilla, Eugenio \\
\hline Paixao Coelho, Jose Augusto & Rex, Tonia \\
\hline Pająk, Paulina & Ribeiro, Maria H \\
\hline Paller, Channing & Ricci, Arianna \\
\hline Palma, Miguel & Robberecht, Harry \\
\hline
\end{tabular}




\begin{tabular}{|c|c|}
\hline Rodriguez-Cueto, Carmen & Silswal, Neerupma \\
\hline Rodriguez-Mateos, Ana & Sinanoglou, Vassilia \\
\hline Romani, Andrea & Singh, Vineet \\
\hline Rose, Peter & Singh, Dhirendra Pratap \\
\hline Ruiz-Aceituno, Laura & Siristatidis, Charalampos \\
\hline Ruperti, Benedetto & Skovsted, Gry Freja \\
\hline Saada, Ann & Slominski, Andrzej \\
\hline Safe, Stephen & Smith, Gaynor Ann \\
\hline Sahrawy, Mariam & Soica, Codruta \\
\hline Sahu, Ravi Prakash & Soveral, Graça \\
\hline Saisho, Yoshifumi & Stagos, Dimitrios \\
\hline Sakurai, Toshihiro & Stefanidou, Maria E. \\
\hline Sandri, Brian J. & Stobdan, Tsering \\
\hline Sanjust, Enrico & Stupka, Nicole \\
\hline Santamaria, Rita & Stushnoff, Cecil \\
\hline Santulli, Gaetano & Subramanian, Veedamali \\
\hline Saraiva, Nuno & Suomela, Jukka-Pekka \\
\hline Sastre, Juan & Suzuki, Yuichiro J. \\
\hline Saturnino, Carmela & Suzuki, Takafumi \\
\hline Savion, Naphtali & Syvänen, Stina \\
\hline Sawicki, Rafal & Szumny, Antoni \\
\hline Schäfer-Korting, Monika & Taira, Junsei \\
\hline Schiraldi, Chiara & Tarrago, Lionel \\
\hline Schlaepfer, Isabel R & Taticchi, Agnese \\
\hline Schmidt, Edward E. & Tavazzi, Barbara \\
\hline Schöttker, Ben & Taylor, Caroline \\
\hline Schröder, Katalin & Teiber, John \\
\hline Schulze, Johannes & Teng, Bo \\
\hline Scicchitano, Pietro & Teschke, Rolf \\
\hline Senbonmatsu, Takaaki & Thierbach, René \\
\hline Seo, Daisuke & Thiyam-Holländer, Usha \\
\hline Serrano, Maria & Thornton, M. Julie \\
\hline Serrano, Núria & Tiidus, Peter \\
\hline Setzer, William N. & Tipple, Trent E. \\
\hline Shao, Baohai & Tokatlidis, Kostas \\
\hline Shaw, Peter & Tomasetti, Marco \\
\hline Sheehy, Paul A. & Tooyama, Ikuo \\
\hline Shi, Vivian Y. & Traustadóttir, Tinna \\
\hline Shiao, Young-Ji & Travers, Jeffrey Bryant \\
\hline Sholkamy, Eman & Tsuji, Petra \\
\hline Shu, Xinhua & Tufarelli, Vincenzo \\
\hline Sieber, Fritz & Ugalde, José Manuel \\
\hline Signorelli, Salvatore Santo & Urfer, Silvan \\
\hline
\end{tabular}


Vaiman, Daniel

Valentová, Kateřina

Valgimigli, Luca

Vallesi, Adriana

Vallini, Giovanni

Van Der Laarse, Willem J.

Van Hecke, Thomas

Vashisth, Tripti

Vaz, Josiana

Vázquez-Medina, José Pablo

Veal, Elizabeth A.

Veeramani, Suresh

Veskoukis, Aristidis S.

Vesoulis, Zachary A.

Vitiello, Peter

Von Knethen, Andreas

Wada, Jun

Wang, Xinkun

Wang, Xinyu

Wang, Horng-dar

Weber, John

Wei, Gang

Weng, Ching-Feng

West, James D.

Wigle, Jeffrey

Wilkinson, Brian
Williams, Christopher C.

Williams, Christopher S.

Wilson, John

Wilson, Mark A

Wojtunik-Kulesza, Karolina A.

Wood, Scott

Woodman, Tim

$\mathrm{Wu}$, Ming-Jiuan

$\mathrm{Wu}$, Li-Chen

Xiaoli, Alus

Xin, Ling

Yi, Tae Hoo

Zachut, Mayak

Zanoli, Luca

Zarrelli, Armando

Zeman, Daniel Meier

Zhang, Pangzhen

Zhang, Hua

Zhou, Huanjiao Jenny

Zlotek, Urszula

Zou, Ping

Zuber, Peter

Zunin, Paola

Żyżelewicz, Dorota

(C) 2019 by the author. Licensee MDPI, Basel, Switzerland. This article is an open access article distributed under the terms and conditions of the Creative Commons Attribution (CC BY) license (http://creativecommons.org/licenses/by/4.0/). 